\title{
SEROPREVALENCIAA SEROVARES DE LEPTOSPIRAS PATÓGENAS EN ALPACAS Y VICUÑAS DE LOS DEPARTAMENTOS DE HUANCAVELICA Y AYACUCHO, PERÚ
}

\author{
Seroprevalence to Pathogenic Leptospira in Alpacas and Vicuñas from \\ Huancavelica and Ayacucho, Peru
}
Raúl Rosadio A. ${ }^{1,2,3}$, Álvaro Véliz A. ${ }^{2}$, Hugo Castillo D. ${ }^{2}$, Katherine Yaya L. ${ }^{2}$, Antony Rodríguez H. ${ }^{2}$, Hermelinda Rivera G. ${ }^{1}$, Jane C. Wheeler ${ }^{2}$

\section{REsumen}

Se realizaron estudios serológicos en 793 alpacas y 195 vicuñas de comunidades campesinas de los departamentos de Huancavelica y Ayacucho, Perú, para detectar anticuerpos específicos para cinco serovares patogénicos (icterohaemorrhagiae, pomona, canicola, wolfii y ballum) mediante la prueba serovar específica de microaglutinación. Se encontró que 89.6\% de las alpacas y 77.4\% de las vicuñas eran seropositivas a uno o más serovares. En alpacas, los anticuerpos tuvieron títulos de 1:100 hasta 1:1600, mayormente con los serovares icterohaemorrhagiae (43.4\%) y pomona (37.8\%); en tanto que en las vicuñas se obtuvo títulos de 1:100 hasta 1:400, reaccionando similarmente a icterohaemorrhagiae (69.2\%) y pomona (8.2\%). Solamente las alpacas mostraron títulos positivos a los serovares canicola (7.8\%) y wolfii (0.6\%). Los resultados indican que los animales han estado expuestos a por lo menos 4 de los 5 serovares estudiados y que las alpacas, por los altos títulos, evidencian historia de infección reciente a los serovares icterohaemorrhagiae y pomona.

Palabras clave: L. icterohaemorrhagiae, L. pomona, alpaca, vicuña, leptospirosis

\section{Abstract}

A serologic study was conducted on 793 alpaca and 195 vicuña sera samples collected in Huancavelica and Ayacucho departments in Peru to detect specific antibodies against five pathogenic leptospira serovars (L. icterohaemorrhagiae, L. pomona, L. canicola, L. wolfii and L. ballum) by the microaglutination test. The survey revealed that $89.6 \%$ of the alpacas and $77.4 \%$ of vicuñas were positive to one or more serovars. In alpacas, the titers varied from 1:100 to 1:1600 reacting mainly to L. icterohaemorrhagiae (43.4\%) and L. pomona (37.8\%), whereas titers among vicuñas varied from 1:100 to 1:400 reacting

${ }^{1}$ Facultad de Medicina Veterinaria, Universidad Nacional Mayor de San Marcos, Lima

${ }^{2}$ CONOPA - Instituto de Investigación y Desarrollo de Camélidos Sudamericanos, Lima

${ }^{3}$ E-mail: rrosadio@gmail.com 
similarly to L. icterohaemorrhagiae (69.2\%) and L. pomona (8.2\%) serovars. The alpacas also had antibodies to $L$. canicola $(7.8 \%)$ and $L$. wolfii $(0.6 \%)$ serovars. These results show that the animals had previous exposure to at least 4 of the 5 serovars tested and the elevated titers to L. icterohaemorrhagiae and L. pomona serovars in alpacas suggest a recent infection event.

Key words: L. icterohaemorrhagiae, L. pomona, alpaca, vicuña, leptospira antibodies

\section{INTRODUCCIÓN}

La infección por bacterias del género Leptospira desencadena procesos subclínicos y clínicos conocidos como leptospirosis en el hombre y en muchas especies de animales silvestres y domésticos (Céspedes et al., 2006). Este agente se mantiene en animales silvestres tipificados como reservorios naturales o portadores, los cuales mantienen el ciclo infectivo contaminando el agua y pastos por la prolongada bacteriuria de los animales infectados (Liceras de Hidalgo et al., 1989; Céspedes, 2005).

La leptospirosis en el Perú se encuentra en las principales ciudades de la costa, sierra y selva, incluyendo, además, ciertas áreas rurales (Céspedes, 2005). En humanos, la infección generalmente se encuentra asociada a estados febriles inespecíficos $\mathrm{y}$, eventualmente, a cuadros clínicos fatales (Céspedes, 2005). En el país, se han aislado varios serovares de casos patológicos en humanos con predominancia de los serogrupos varrilla e icterohemorrhagiae (Céspedes et al., 2006). Los aislados en humanos proceden, aunque en menor cantidad, de los departamentos de Huancavelica y Ayacucho (Liceras de Hidalgo et al., 1989; Céspedes et al., 2006), pero con presencia de anticuerpos para cinco serovares en Huancavelica y 12 en Ayacucho.

Por otro lado, la leptospirosis es responsable en muchos países latinoamericanos de cuadros patológicos reproductivos en bovinos y porcinos (Cachata et al., 2008). El rol patogénico de esta bacteria en el Perú no está bien esclarecido, pero se cree que los bovi- nos padecen similares patologías reproductivas (Céspedes, 2005). Los escasos estudios serológicos realizados en el país evidencian una prevalencia de $20.6 \%$ para bovinos de valles costeros y $23.6 \%$ en animales procedentes de valles interandinos (Liceras de Hidalgo et al., 1989).

La epidemiología de la infección en animales domésticos en zonas altoandinas se encuentra pobremente elucidada. Estudios serológicos realizados en 810 alpacas de la región del altiplano puneño indican una prevalencia de $6.5 \%$ para siete serovares, pero con una mayor frecuencia (79.2\%) para el serovar L. pomona (Herrera et al., 2000). En una reciente investigación realizada en 208 vicuñas (silvestres y en cautiverio), 574 alpacas y 43 llamas procedentes de comunidades campesinas de Salinas y Aguada Blanca (Arequipa) se determinó la presencia de bajos títulos de anticuerpos en vicuñas para los serovares copenhageni (3.4\%), pomona (1.4\%) y autumnalis (1\%), mientras que las alpacas y llamas mostraron similares anticuerpos, en altos títulos, predominantemente a los serovares de pomona (19.4\%) y autunmalis (13.8\%) (Ortega et al., 2009). Por otro lado, en alpacas de zonas colindantes y perteneciente a la provincia de Canchis, Cusco, se detectó una mayor prevalencia para los serovares pomona (49\%), icterohemorragiae (47\%) y canicola (19\%) (Rosadio et al., 2003).

El presente estudio reporta resultados de análisis serológicos realizados en 793 alpacas y 195 vicuñas procedentes de áreas rurales de los Andes centrales (departamentos de Huancavelica y Ayacucho) frente a cinco serovares: icterohemorragiae, pomona, canicola, ballum y wolfii. 
Materiales y MÉtodos

\section{Muestreo de Animales}

El muestreo de animales fue realizado como parte de ejecución del proyecto "Programa de manejo sanitario de camélidos en comunidades alto andinas del área de influencia del proyecto Perú LNG”, desarrollado por el Instituto de Investigación y Desarrollo de Camélidos Sudamericanos (CONOPA) entre diciembre de 2008 a octubre de 2009.

Se colectó muestras de sangre a 793 alpacas procedentes de 66 comunidades campesinas de los departamentos de Huancavelica y Ayacucho, Perú. Para el estudio, se escogieron establecimientos alpaqueros con más de 100 animales, muestreando entre 15 a 20 animales por establecimiento, preferentemente hembras, escogidos al azar, y en aparente buen estado de salud.

Asimismo, se colectó 195 muestras de vicuñas procedentes de tres comunidades campesinas y de una área multicomunal en el departamento de Huancavelica. Las muestras se recolectaron durante los "chaccus" oficiales programados y autorizados por el Ministerio de Agricultura (109-2009-AGDGFFS-DGEFFS).

Las muestras de las alpacas fueron obtenidas en los meses de febrero y marzo, y de las vicuñas en julio de 2009. Las muestras de sangre fueron extraídas de la vena yugular y los sueros se obtuvieron por centrifugación y almacenados a $-20^{\circ} \mathrm{C}$.

\section{Prueba de Aglutinación Microscópica}

La detección de anticuerpos serovares específicos se hizo mediante la técnica de aglutinación microscópica, siguiendo el protocolo disponible en el Laboratorio de Microbiología de la Facultad de Medicina Veterinaria, Universidad Nacional de San Marcos, de acuerdo al Manual de la Organización Mundial de Sanidad Animal (OIE, 1996).
En la prueba se empleó los cinco serovares más prevalentes en animales domésticos en el país: icterohemorragaie, pomona, canicola, wolfii y ballum. Las diluciones de trabajo, en soluciones fisiológicas estériles de los sueros fueron desde 1/50 hasta $1 / 6400$. Inicialmente, los sueros fueron sometidos a prueba tamiz para descartar sueros negativos $(<1 / 100)$, y continuar analizando los positivos $(\geq 1 / 100)$. La lectura de aglutinación se llevó a cabo midiendo la reducción del 50\% de las leptospiras libres no aglutinadas utilizando microscopio de campo oscuro y considerando seropositividad a aquellos sueros con reacciones positivas en diluciones $\geq 1 / 100$.

\section{Resultados y Discusión}

El 89.6\% ( $\mathrm{n}=711)$ de las alpacas reaccionaron positivamente a cuatro serovares patogénicos de leptospira; especialmente a los serovares L. icterohemorrhagiae (43.4\%) y L. pomona (37.8\%) (Cuadro 1). Asimismo, el $77.4 \%(n=151)$ de las vicuñas reaccionaron a los serovares $L$. icterohaemorrhagiae (69.2\%) y L. pomona (8.2\%) (Cuadro 1).

En las alpacas, los títulos variaron desde 1:100 hasta 1:1600, pero la mayoría (73.3\%) de los animales presentó títulos entre 1:100 y 1:200 (Cuadro 2). Las vicuñas, sin embargo, solo llegaron a alcanzar títulos de 1:400 (Cuadro 3).

El Perú es depositario de la mayor población de alpacas en el mundo y la agroindustria que gira alrededor de los camélidos sudamericanos (CSA) es una actividad muy particular e ideal para las condiciones de los sistemas de producción animal altoandinos. Sin embargo, la crianza y explotación de estas especies se encuentra permanentemente amenazada por problemas sanitarios, tales como problemas reproductivos con elevadas pérdidas embrio-narias y abortos (Novoa y Flores, 1991). En el Perú, los casos de abortos infecciosos en alpacas 
Cuadro 1. Resultados de la prueba de microaglutinación para la detección de anticuerpos serovares específicos contra Leptospira en alpacas y vicuñas de Huancavelica y Ayacucho (2009)

\begin{tabular}{|c|c|c|c|}
\hline \multirow{2}{*}{ Especie } & \multirow{2}{*}{ Serovares } & \multicolumn{2}{|c|}{ Reactores } \\
\hline & & $\mathrm{n}$ & $\%$ \\
\hline \multirow{6}{*}{$\begin{array}{l}\text { Alpaca } \\
(\mathrm{n}=793)\end{array}$} & L. icterohemorrhagiae & 344 & 43.4 \\
\hline & L. pomona & 300 & 37.8 \\
\hline & L. canicola & 62 & 7.8 \\
\hline & L. wolfii & 05 & 0.6 \\
\hline & L. ballum & 0 & - \\
\hline & Total & 711 & 89.6 \\
\hline \multirow{6}{*}{$\begin{array}{l}\text { Vicuña } \\
(\mathrm{n}=195)\end{array}$} & L. icterohaemorrhagiae & 135 & 69.2 \\
\hline & L. pomona & 16 & 8.2 \\
\hline & L. canicola & 0 & - \\
\hline & L.wolfii & 0 & - \\
\hline & L. ballum & 0 & - \\
\hline & Total & 151 & 77.4 \\
\hline
\end{tabular}

Cuadro 2. Títulos de anticuerpos y seroprevalencia de leptospirosis en 793 alpacas de Huancavelica y Ayacucho (2009)

\begin{tabular}{|c|c|c|c|c|c|c|c|}
\hline \multirow{2}{*}{ Serovares } & \multirow{2}{*}{$1 / 100$} & \multirow{2}{*}{$1 / 200$} & \multirow{2}{*}{$1 / 400$} & \multirow{2}{*}{$1 / 800$} & \multirow{2}{*}{$1 / 1600$} & \multicolumn{2}{|c|}{ Total } \\
\hline & & & & & & $\mathrm{n}$ & $\%$ \\
\hline icterohaemorrhagiae & 20 & 230 & 73 & 19 & 2 & 344 & 43.4 \\
\hline pomona & 180 & 84 & 31 & 4 & 1 & 300 & 37.8 \\
\hline canicola & 52 & 10 & 0 & 0 & 0 & 62 & 7.8 \\
\hline wolfii & 5 & 0 & 0 & 0 & 0 & 5 & 0.6 \\
\hline ballum & 0 & 0 & 0 & 0 & 0 & 0 & 0 \\
\hline \multirow{2}{*}{ Total } & 257 & 324 & 104 & 23 & 3 & 711 & \\
\hline & 32.4 & 40.9 & 13.1 & 2.9 & 0.4 & & 89.6 \\
\hline
\end{tabular}


Cuadro 3. Títulos de anticuerpos y seroprevalencia de leptospirosis en 195 vicuñas de Huancavelica y Ayacucho (2009)

\begin{tabular}{|c|c|c|c|c|c|c|c|}
\hline \multirow{2}{*}{ Serovares } & \multirow{2}{*}{$1 / 100$} & \multirow{2}{*}{$1 / 200$} & \multirow{2}{*}{$1 / 400$} & \multirow{2}{*}{$1 / 800$} & \multirow{2}{*}{$1 / 1600$} & \multicolumn{2}{|c|}{ Total } \\
\hline & & & & & & $\mathrm{n}$ & $\%$ \\
\hline icterohaemorrhagiae & 97 & 34 & 4 & 0 & 0 & 135 & 69.2 \\
\hline pomona & 16 & 0 & 0 & 0 & 0 & 16 & 8.2 \\
\hline canicola & 0 & 0 & 0 & 0 & 0 & 0 & 0 \\
\hline wolfii & 0 & 0 & 0 & 0 & 0 & 0 & 0 \\
\hline ballum & 0 & 0 & 0 & 0 & 0 & 0 & 0 \\
\hline \multirow{2}{*}{ Total } & 113 & 34 & 4 & 0 & 0 & 151 & \\
\hline & 57.9 & 17.4 & 2.0 & 0 & 0 & & 77.4 \\
\hline
\end{tabular}

han sido atribuidos a infecciones por Brucella mellitensis (Novoa y Flores, 1991) y Neospora caninum (Serrano-Martínez et al., 2007), pero se sospechan de algunos serovares leptospirales e infección pestiviral (Rosadio et al., 2003).

El análisis serológico evidencia que la vicuña y la alpaca, especies genéticamente relacionadas (Wheeler, 1995), son aparentemente susceptibles a determinados serovares patogénicos - L. pomona e icterohemorrhagiae -. La biología de la infección en estos animales es obscura, pero existe un reporte de una infección fatal en guanaco (Hodgin, 1984) y presencia de elevados títulos a $L$. grippotyphosa asociados con abortos en un hato de llamas americanas (Pugh et al., 1995). La prevalencia de anticuerpos contra algunos de los serovares en estudio fue alta con respecto a lo reportado en bovinos de zonas andinas del sur (Cachata et al., 2008) y en cuatro establecimientos alpaqueros del departamento de Puno (Herrera et al., 2000).

Los resultados evidencian seroreacciones en alpacas y vicuñas, particularmente a los serovares icterohaemorrhagiae y pomona en tasas similares identificadas en alpacas de la provincia de Canchis, Cuzco y
Santa Lucía, Puno (Rosadio et al., 2003; Santos et al., 2009) y a los CSA domésticos y silvestres de varias regiones de la Argentina (Llorente et al., 2002). La alta prevalencia, particularmente al serovar pomona en las vicuñas muestreadas en el estudio, difiere de lo detectado en 208 vicuñas de la reserva de Salinas y Aguada Blanca (Ortega et al., 2009). Estos autores reportan una baja prevalencia (7.2\%) pero con altos títulos a los serovares de autumnalis y pyogenes y con bajos títulos a los serovares pomona, copenhageni y bratislava.

A pesar de la disponibilidad de datos sobre prevalencia serológica, muy poco se sabe sobre la epidemiología y la biología de la infección de la leptopirosis en CSA en el Perú. Llama particularmente la atención la seropositividad a los serovares icterohaemorrhagiae y pomona en ambas especies. La presencia de anticuerpos a cuatro serovares en alpacas indica la exposición a serovares que utilizan roedores como animales portadores (icterohaemorrhagiae), rumiantes andinos (pomona), perros (canicola) y murciélagos (wolfii). Todos estos animales coexisten en áreas altoandinas peruanas y bien podrían ser las posibles fuentes de infección para los CSA. 
La leptospirosis esta ampliamente distribuida en el Perú, y a nivel andino, se han aislado, en humanos y animales, varios serovares patógenos, entre ellos: icterohaemorrhagiae, pomona, canicola (Liceras de Hidalgo et al., 1989). Por otro lado, el serovar pomona ha sido confirmado en bovinos y cerdos en los dos departamentos estudiados y los trabajos de seroprevalencia muestran un alto porcentaje de seropositivos, particularmente a icterohaemorrhagiae en pobladores del departamento de Ayacucho (Vargas-Cuba et al., 2008).

Se desconoce la biología de la infección leptospiral en los camélidos pero la elevada prevalencia de anticuerpos encontrados en alpacas para los serovares bajo estudio sugiere no solamente historia de infección (bajos títulos), pero sobretodo exposición reciente, principalmente por parte de las alpacas al serovar pomona (elevados títulos). La seropositividad, sugiere, además, que estos animales podrían estar actuando como diseminadores de la bacteria al medio ambiente, por lo que se recomienda elucidar la asociación de leptospira y enfermedad clínica incluyendo la posible habilidad abortigénica en los CSA.

\section{LiTERATURA Citada}

1. Cachata S, Suárez F, Huanca W, Rivera H. 2008. Prevalencia de anticuerpos contra Leptospira sp en dos predios de Puno. Rev Inv Vet, Perú 19: 187-191.

2. Céspedes M. 2005. Leptospirosis: enfermedad reemergente. Rev Per Med Exp Salud Pública 22(4): 1-18.

3. Céspedes M, Balda L, Gonzáles D, Tapia R. 2006. Situación de la leptospirosis en el Perú. 1994-2004. Rev Per Med Exp Salud Pública 23(1): 56-66.

4. Herrera Carpio JP, Vasconcellos SA, Morais ZM, Ferreira F. Sakamoto SM, Ferreira Neto JS, Pinheiro SR. 2000. Seropositividad para leptospirose em alpacas criadas no altiplano peruano. Puno, Peru. Arq Inst São Paulo 67: 171-176.

5. Liceras de Hidalgo J, Valdivia Paz Soldán S, Higuchi E. 1989. Leptospirosis en Perú. Anales del Seminario Nacional de Zoonosis y Enfermedades de Transmisión Alimentaria. Lima: Ministerio de Salud/OPS/Concytec. p 7-20.

6. Llorente P, Leoni L, Martínez Vivot M. 2002. Leptospirosis en camélidos sudamericanos. Estudio de prevalencia serológica en distintas regiones de Argentina. Arch Med Vet 34(1): 59-68.

7. Hodgin C. 1984. Leptospirosis and coccidial infection in a guanaco. JAVMA 11: $1442-1444$

8. Novoa C, Flores A. 1991. Producción de rumiantes menores: alpacas. Lima, Perú: RERUMEN. 358 p.

9. Ortega LM, Risco V, Arnaiz I, GarcíaPeña F, Rosadio R, Castillo $H$, Wheeler J, Hoces D. 2009. Evaluación del impacto sanitario de los nuevos sistemas de manejo en la población de vicuña del Perú. En: López D, Vendrell E (eds). Investigación en agricultura para el desarrollo. Barcelona: Red de Investigación en Agricultura para el Desarrollo. p 182-187.

10. [OIE] Office International des Epizooties. 1996. Leptospirosis. In: Manual of standards for diagnostic tests and vaccines. Paris: OIE. p 198-201.

11. Pugh DG Wright J, Rowe S. 1995. Serological response of llamas to commercially prepared leptospirosis vaccine. Small Rum Res 17: 193-196.

12. Rosadio R, Rivera H, Chávez A, Serrano E. Quispe R, Yaya C. 2003. Seroprevalencia de agentes abortigénicos en alpacas de la provincia de Canchis (Cusco, Perú). En: Memoria III Congreso Mundial sobre Camélidos. Potosí, Bolivia. p 863-872.

13. Santos Y, Suárez F, Rivera H, Huanca W, Cárdenas O, Camacho J. 2009. Seroprevalencia de leptospirosis en alpacas de Quimsachata, Puno. Rev Inv Vet, Perú 20: 108-113. 
14. Serrano-Martínez E, CollantesFernández E, Chávez-Velásquez A, Rodríguez-Bertos A, Casas-Astos E, Risco-Castillo V, Rosadio-Alcántara R, Ortega-Mora LM. 2007. Evaluation of Neospora caninum and Toxoplasma gondii infections in alpaca (Vicugna pacos) and llama (Lama glama) aborted fetuses from Peru. Vet Parasitol 150: 39-45.
15. Vargas-Cuba F, García-Apaico V, Céspedes M, Palomino-Enciso M, AyalaHuaytalla T. 2008. Seroprevalencia y factores asociados con leptospirosis en pacientes con síndrome febril en Ayacucho, Perú. Rev Per Med Exp Salud Pública 25(2): 190-194.

16. Wheeler JC. 1995. Evolution and present situation of the South American Camelidae. Biol J Linn Soc 54: 271-295. 